\title{
An index of inflammatory activity in patients with Crohn's disease
}

\author{
P A M VAN HEES,* PH VAN ELTEREN, H J J VAN LIER, AND J H M VAN \\ TONGEREN
}

From the Department of Medicine, Division of Gastroenterology, St. Radboud Hospital, University of Nijmegen, and the Statistical Support Centre, University of Nijmegen, The Netherlands

SUMMARY An objective and quantitative standard of inflammatory activity for patients with Crohn's disease was developed. This Activity Index (AI) was derived from data of 63 patients with Crohn's disease who had been submitted to a total of 85 clinical examinations. On the basis of 18 predictor variables three physicians gave an overall evaluation of the severity of inflammatory activity in each patient. Stepwise multiple regression analysis was used to investigate which combination of variables contributed most to the overall evaluation. The combination of the following nine variables gave a very good correlation $(r=0.95)$ with the overall evaluation: serum albumin, ESR, body weight related to length, abdominal mass, sex, temperature, stool consistency, bowel resection, and extraintestinal symptoms related to Crohn's disease. This combination of variables expressed in a score that was used as an activity index proved to be very useful in the assessment of disease activity and of the effect of therapy. Index values below 100 are associated with inactive disease, values between 100 and 150 can be regarded as indicating slight inflammatory activity, values between 150 and 210 as indicating moderate, and values above 210 as indicating severe-to-very-severe inflammatory activity.

Crohn's disease is a condition which encompasses a wide variety of complaints and symptoms. These may be related to the inflammatory activity, but even when there is little activity there may be considerable subjective complaints-for example, of abdominal pain as a result of the presence of an inactive fibrous stricture. This diversity of the causes of the complaints and the lesions found greatly impedes exact evaluation of the effect of various types of therapeutic medication on the inflammatory activity. During the preparations for a prospective study which was to establish the effect of various types of therapeutic medication on the inflammatory activity in Crohn's disease, the need arose for an optimally objective and quantifiable standard of inflammatory activity. The Crohn's Disease Activity Index (CDAI) described by Best et al. ${ }^{1}$ has, we believe, several disadvantages. With the aid of data from a retrospective study, we therefore attempted to develop a more suitable activity index-that is, an optimally objective and reproducible quantitative

*Address for correspondence: P A M van Hees, Department of Medicine, Division of Gastroenterology, St. Radboud Hospital, Nijmegen, The Netherlands.

Received for publication 25 October 1979 standard of inflammatory activity-for patients with Crohn's disease.

Table 1 Frequency distribution of some variables over 85 clinical examinations in 63 patients with Crohn's disease, during which data used in activity index were collected

\begin{tabular}{ll}
\hline & Examinations \\
\cline { 2 - 2 } & $(n o)$ \\
\hline Sex & \\
Male & 36 \\
Female & 49 \\
Age (yr) & 24 \\
$<25$ & 50 \\
$25-50$ & 11 \\
50 and over & 24 \\
Previous operations & 59 \\
Ileocaecal resection & 58 \\
Localisation of Crohn's disease & 5 \\
Small intestine & 3 \\
Small intestine and colon & \\
Colon & 42 \\
None (after resection) & 32 \\
Therapeutic medication & 4 \\
None & 3 \\
Salicylazosulphapyridine & 4 \\
Salicylazosulphapyridine and prednisone & \\
Prednisone & \\
Antibiotics & \\
\hline
\end{tabular}




\section{Methods}

\section{PATIENTS}

The retrospective study covered the data on 63 patients with Crohn's disease who, during the period 1963-75, had been submitted to a total of 85 clinical examinations.

Crohn's disease was diagnosed on the basis of macroscopic and histological lesions in resected specimens and/or typical features found at radiological examination of the colon and small intestine. Some data on these patients are listed in Table 1. None of these patients was using obstipants or an elemental diet. Patients who had undergone extensive bowel resections (more than $1 \mathrm{~m}$ of small intestine or more than one-third of the colon) and patients with an ileostomy or colostomy were not included in the study. The study considered only those hospital periods in which the 18 variables listed in Table 2 had all been collected. The Quetelet-index

Table 2 Independent variables used in development of activity index

\begin{tabular}{|c|c|c|}
\hline \multicolumn{2}{|c|}{ Variable } & \multirow{2}{*}{$\frac{\text { Unit/code }}{\text { Number of defaecations per day }}$} \\
\hline$x_{1}$ & Rate of defaecation & \\
\hline$x_{2}$ & Stool consistency & $\begin{array}{l}1=\text { well-formed; } 2=\text { soft, vari- } \\
\text { able; } 3=\text { watery }\end{array}$ \\
\hline $\mathbf{x}_{3}$ & Macroscopic blood in faeces & $1=$ no $; 2=$ moderate; $3=$ much \\
\hline$x_{4}$ & Macroscopic mucus in faeces & $1=$ no $; 2=$ yes \\
\hline$x_{3}$ & Temperature & Centigrade \\
\hline$x_{6}$ & Abdominal mass & $\begin{array}{l}1=\text { no; } 2=\text { dubious; } 3=\text { dia- }- \\
\text { meter }<6 \mathrm{~cm} ; 4=\text { diameter } \\
6-12 \mathrm{~cm} ; 5=\text { diameter }>12 \\
\mathrm{~cm}\end{array}$ \\
\hline $\mathbf{x}_{\mathbf{7}}$ & (Sub)ileus symptoms & $\begin{array}{l}1=\text { no; } 2=\text { slight } ; 3=\text { moderate; } \\
\quad 4=\text { severe }\end{array}$ \\
\hline$x_{8}$ & Anal/perianal lesions & $1=$ no $; 2=$ yes \\
\hline$x_{9}$ & Extraintestinal lesions & $1=$ no $; 2=$ yes \\
\hline$x_{10}$ & $\begin{array}{l}\text { Bowel resection for Crohn's } \\
\text { disease }\end{array}$ & $1=$ no; $2=$ yes \\
\hline$x_{11}$ & Quetelet index $\left(\mathbf{W} / \mathbf{H}^{2}\right)$ & $\begin{array}{l}\mathrm{W}=10 \times \text { body weight in } \mathrm{kg} \\
\mathrm{H}=\text { height in } \mathrm{m}\end{array}$ \\
\hline$x_{12}$ & ESR & $\mathrm{mm}$ after $1 \mathrm{~h}$ \\
\hline$x_{13}$ & Haemoglobin concentration & $\mathrm{mmol} / \mathrm{l}$ \\
\hline$x_{14}$ & Total serum protein level & $\mathrm{g} / \mathrm{l}$ \\
\hline$x_{15}$ & Serum albumin level & $\mathrm{g} / 1$ \\
\hline$x_{16}$ & Serum $\gamma$-globulin level & g/l \\
\hline$x_{17}$ & Sex & $1=$ male $; 2=$ female \\
\hline $\mathrm{X}_{18}$ & Stool weight & $\begin{array}{l}\mathrm{g} / 24 \mathrm{~h} \text { (mean of } 5 \text { consecutive } \\
\text { days) }\end{array}$ \\
\hline
\end{tabular}

relates body weight to body height. ${ }^{23}$ Fissures, fistulae, and perianal abscesses were taken into account as anal and perianal lesions. Arthritis, stomatitis (apthosa), erythema nodosum, episcleritis, iritis, and iridocyclitis were taken into account as extraintestinal lesions related to Crohn's disease.

The total serum protein concentration was determined with the aid of the biuret reagent; serum albumin and $\gamma$-globulin concentrations were determined by cellulose acetate electrophoresis. Reference (normal) values are: total protein $65-75 \mathrm{~g} / \mathrm{l}$; albumin 45-55 g/l, and $\gamma$-globulin 6-12 g/l; haemoglobin level $8.7-10.7 \mathrm{mmol} / \mathrm{l}$ for males and 7.9-9.8 $\mathrm{mmol} / \mathrm{l}$ for females.

\section{STATISTICAL METHODS}

The series of values of the variables listed in Table 2, obtained in one examination, will be referred to as a measurement set. Of each of the 85 measurement sets, two prints were made. These prints were laid out in random order, and three specialists in the department of gastroenterology who were involved in the treatment of patients with Crohn's disease were asked, independently and without knowledge of the patient's identity, to rate the activity of the inflammation from each measurement set. The following activity score was used: $1=$ not active, $2=$ slight, $3=$ moderate, $4=$ severe, and $5=$ very severe inflammatory activity. Next, an effort was made to establish whether sufficient agreement existed between the two ratings of the same measurement set by each of the specialists. This agreement was found to be very good. The two activity scores per specialist were identical for 75,78 , and 81 of the 85 measurement sets, respectively. The difference between the two activity scores assigned to the same measurement set by one specialist never exceeded 1 point. In an effort to establish whether the activity scores assigned by the three specialists were in agreement, the correlation coefficients between the specialists' mean activity scores were calculated. These correlation coefficients were $0.92,0.88$, and 0.90 , respectively. In view of these results it was considered justifiable to summarise the specialists' scores for each measurement set in a single figure. Each measurement set was rated twice by each specialist, and the sum of their activity scores had consequently to range from a minimum of 6 points to a maximum of 30 points. This sum, multiplied by factor 10 for convenience, was called the (physician's) activity rating (y) and regarded as a standard of the subjective view of the three specialists on the inflammatory activity of the disease. This activity rating can range from 60 to 300 points. The boundaries between the activity classes were defined as indicated in Table 3. On the basis of this classification, disease activity was absent for $8 \%$, slight for

Table 3 Boundaries between Crohn's disease activity classes, based on subjective rating by three specialists

\begin{tabular}{ccl}
\hline $\begin{array}{l}\text { Mean of six } \\
\text { activity scores }(a)\end{array}$ & $\begin{array}{l}\text { Physician's rating } \\
\text { of activity }(y)\end{array}$ & $\begin{array}{l}\text { Activity of Crohn's } \\
\text { disease }\end{array}$ \\
\hline $\mathrm{a} \leq 1 \cdot 5$ & $\mathrm{y} \leq 90$ & No \\
$1 \cdot 5<\mathrm{a} \leq 2 \cdot 5$ & $90<\mathrm{y} \leq 150$ & Slight \\
$2 \cdot 5<\mathrm{a} \leq 3.5$ & $150<\mathrm{y} \leq 210$ & Moderate \\
$\mathrm{a}>3.5$ & $\mathrm{y} \leq 210$ & (Very) severe \\
\hline
\end{tabular}


$28 \%$, moderate for $26 \%$, and severe-to-very-severe for $38 \%$ of the measurement sets.

The purpose was to construct an activity index (AI) on the basis of the variables $x_{1}, x_{2}$, etc. of Table 2, used also in the subjective rating of the activity by the three specialists. For practical reasons we decided to choose as AI a linear function $\hat{y}=$ $b_{0}+b_{1} x_{1}+b_{2} x_{2}+\ldots+b_{k} x_{k}$ of a number $(k)$ of the variables mentioned. The intention was then so to determine the number of variables and the constants $b_{0}, b_{1} \ldots, b_{k}$ that the AI sufficiently approximated the views of the three specialists on the 85 measurement sets (expressed in activity rating y). Multiple regression analysis was applied for this purpose.

Use was made of the stepwise version of the Multiple Regression Programme of the SPSS (Statistical Package for the Social Sciences). ${ }^{4}$ In this study we refrained from adding yet another variable when the multiple correlation coefficient $\mathrm{R}$-that is, the correlation coefficient between activity rating $(y)$ and $\mathrm{AI}(\hat{\mathrm{y}})$-exceeded 0.95 .

By inserting the values of the variables $x_{1}, \ldots, x_{k}$ found for an individual patient into the formula obtained, the AI as a standard of the activity of Crohn's disease can be obtained for that patient.

Table 4 lists these variables in the order of their inclusion in the regression equation; consequently, the successive (residual) standard deviations $\left(\operatorname{SD}_{\text {res }}(\mathrm{y})\right.$ ) indicate how the accuracy of the index increases upon inclusion of each new variable in the regression equation.

The coefficients $b_{0}$ and $b_{1}$ shown in Table 4 refer to the cellulose acetate electrophoresis (cae) method used in our laboratory for determining serum albumin. Other laboratories may obtain different serum albumin values in the same blood sample using cae or another method, which may be apparent from different reference values. Radial immunodiffusion (rid) according to Mancini is considered to be a more specific and less laboratory dependent method for determining serum albumin. From 80 paired determinations of serum albumin according to the cae and the rid method (M-Parigen $ß$ albumin/ albumin; Behring Institut, Germany) in our laboratory the following relation between the cellulose acetate electrophoresis values $\left(x_{1}\right)$ and the radial immunodiffusion values $\left(\mathrm{x}_{1}{ }^{\prime}\right)$ was established: $\mathrm{x}_{1}=9 \cdot 4+0 \cdot 90 \mathrm{x}_{1}{ }^{\prime} \pm 3.7$ (residual S.D), resulting in coefficients $b_{o}{ }^{\prime}=-261$ and $b_{1}{ }^{\prime}=-4.93$ in the formula for the AI with respect to rid determined serum albumin. If the reference values of serum albumin in another laboratory are different from our reference values we recommend making a series of paired measurements of serum albumin (with a range of about 20-55 g/l) with both the rid method $\left(\mathrm{x}_{1}{ }^{\prime}\right)$ and the method used in that laboratory $\left(\mathrm{x}_{1}{ }^{\prime \prime}\right)$ and to compute the least squares regression equation of $x_{1}$ " with respect to $\mathrm{x}_{1}{ }^{\prime}: \mathrm{x}_{1}{ }^{\prime \prime}=\mathrm{c}_{\mathrm{o}}+\mathrm{c}_{1} \mathrm{x}_{1}{ }^{\prime} \pm \mathrm{s}$ (residual SD). The coefficients with respect to $x_{1}{ }^{\prime \prime}$ then are: $b_{o}{ }^{\prime \prime}=$ $-261+.4 .93 c_{0} / c_{1}$ and $b_{1}{ }^{\prime \prime}=-4.93 / c_{1}$.

\section{EVALUATION OF ACTIVITY INDEX (AI)}

AND CDAI

The retrospective data were analysed also by three gastroenterologists from three other institutions, not involved in the development of the AI, to see if there was a correlation between their evaluation scores and the AI. The two activity scores per gastroenterologist were identical for 78,60 , and 65 of the 85 measurement sets, respectively. Except for one measurement set the difference between the two activity scores assigned to the same measurement set by one gastroenterologist never exceeded 1 point. The correlation coefficients between the gastroenterologists' mean

Table 4 Activity index $A I=b_{0}+\Sigma_{\mathrm{i}} b_{\mathrm{i}} x_{\mathrm{i}}$

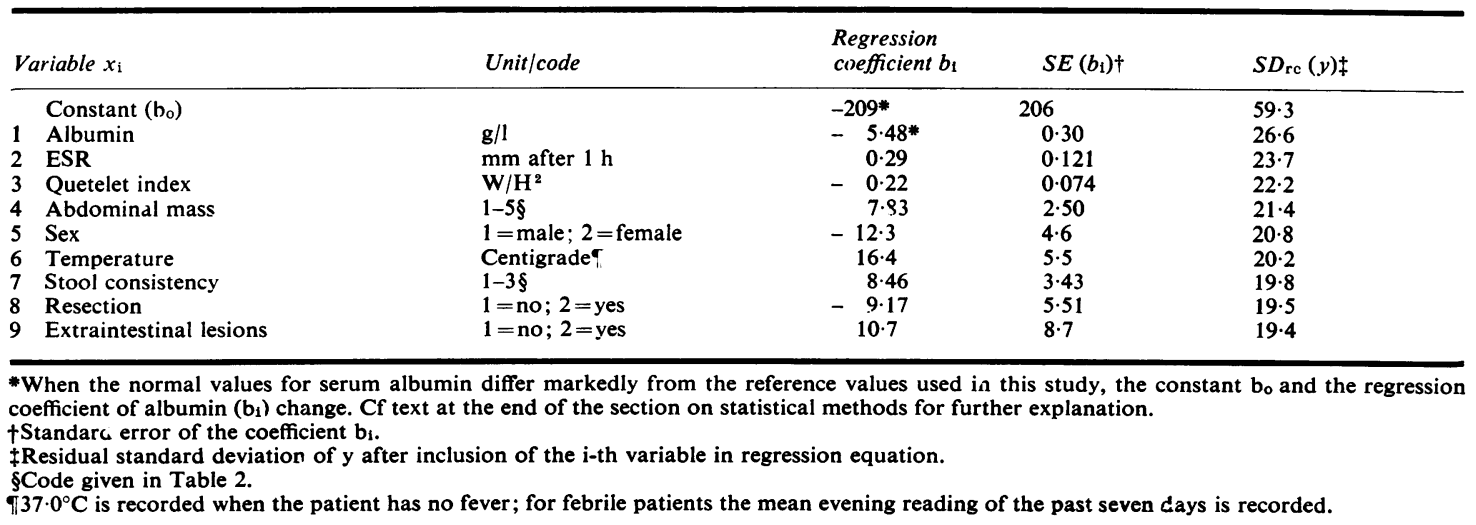


activity scores were $\mathbf{0} \cdot 81,0 \cdot 80$, and $0 \cdot 87$, respectively.

In order to gain an impression of the values of the AI and CDAI in normal individuals, both indices were determined in 20 healthy volunteers.

During the period June 1977 to April 1978, the inflammatory activity (expressed in activity score 1 through 5) was rated in 34 clinical patients with Crohn's disease (mostly in an active stage) and 30 outpatients with the same disease (in remission or in course of treatment) who were examined by one of us. This was done on the basis of the history, physical examination, laboratory findings, and (if recently performed) radiological examination. The AI and CDAI were then calculated. In 36 of these 64 patients the disease was localised in the small intestine; in 25 it was localised both in the small intestine and in the colon; in three patients, there was no longer any localisation of Crohn's disease after a resection. A total of 24 of these 64 patients had undergone a resection (usually ileocaecal resection) in the past.

In order to establish whether the stay in hospital influenced AI and CDAI, both indices were calculated in 16 of the 64 patients at the end of a hospital period and at the first outpatient follow-up two weeks after discharge from hospital.

In the same 16 patients, finally, the AI and CDAI were determined before and during therapeutic medication (one to six months later). An attempt was made to establish whether the change in AI and CDAI corresponded with the physician's assessment of the effect of therapy, so far as this became manifest in a change in the physician's activity score. In all cases this assessment of inflammatory activity was made before AI and CDAI were calculated.

\section{Results}

ACTIVITY INDEX (AI)

It proved to be possible to develop, on the basis of nine variables, an activity index which attained a correlation coefficient of $95 \%$ with the corresponding physician's rating (activity rating y). Table 4 lists these variables and the constants $\left(b_{i}\right)$ required for calculation of the AI. Table 5 gives an example of the calculation of the AI for a given patient.

The physician's rating $(y)$ has a standard deviation of 59.3 points. In relation to an index based solely on the serum albumin level, only a residual standard deviation (SDres $(y))$ of 26.6 points remains (Table 4). The other variables also cause an unmistakable further reduction of the $\operatorname{SD}_{\text {res }}(y)$ : by $27 \%$ to $19 \cdot 4$ points.

Figure 1 shows the relation between $\mathrm{AI}$ and physician's rating. It also indicates the boundaries
Table 5 Example of calculation of AI for a given patient

\begin{tabular}{|c|c|c|c|}
\hline $\begin{array}{l}\text { Serum albumin: } 29 \mathrm{~g} / 1 \\
\text { ESR: } 45 \mathrm{~mm} \\
\text { Weight: } 65 \mathrm{hg} \text {; height: } 1 \cdot 78 \mathrm{~m} \\
650\end{array}$ & $\begin{array}{l}29 \\
45\end{array}$ & $\begin{array}{l}\times-5.48= \\
\times \quad 0.29=\end{array}$ & $\begin{array}{r}-158 \cdot 92 \\
13.05\end{array}$ \\
\hline Quetelet index: $\overline{1.78 \times 1.78}=205$ & 205 & $\times-0.22=$ & $-45 \cdot 1$ \\
\hline $\begin{array}{l}\text { Abdominal mass: absent } \\
\text { Sex:female* } \\
\text { Temperature: } 37 \cdot 0^{\circ} \mathrm{C} \\
\text { Stool consistency: soft* } \\
\text { Resection: yes* } \\
\text { Extraintestinal lesions: no* }\end{array}$ & $\begin{array}{c}1 \\
2 \\
37 \cdot 0 \\
2 \\
2 \\
1\end{array}$ & $\begin{array}{l}\times 7 \cdot 83= \\
\times-12 \cdot 3= \\
\times 16 \cdot 4= \\
\times 8 \cdot 46= \\
\times-9 \cdot 17= \\
\times 10 \cdot 7=\end{array}$ & $\begin{array}{c}7 \cdot 83 \\
-24 \cdot 6 \\
606 \cdot 8 \\
16 \cdot 92 \\
-18 \cdot 34 \\
10 \cdot 7\end{array}$ \\
\hline & \multicolumn{2}{|c|}{ Constant $\left(b_{0}\right)$} & $\begin{array}{l}408 \cdot 34 \\
-209\end{array}$ \\
\hline & \multicolumn{2}{|l|}{ AI } & 199 \\
\hline
\end{tabular}

*For codes see Table 2.

of the activity classes (no, slight, moderate, anh severe-to-very-severe inflammatory activity), botd according to the AI (i) and according to the physician's rating (y) (cf Table 3). To avoid patients with no or very slight activity according to the AI from being erroneously included in the slight activity class, the boundary between 'no' and 'slight' activity was set at $A I=100$. An $A I$ in the range 100-150 can be regarded as indicating slight inflammatory activity, an AI in the range $150-210$ as indicating moderate, and an $\mathrm{Al}>210$ as indicating severe-to-very-severe inflammatory activity.

Figure 1 shows that the vast majority $(78 \%)$ of the

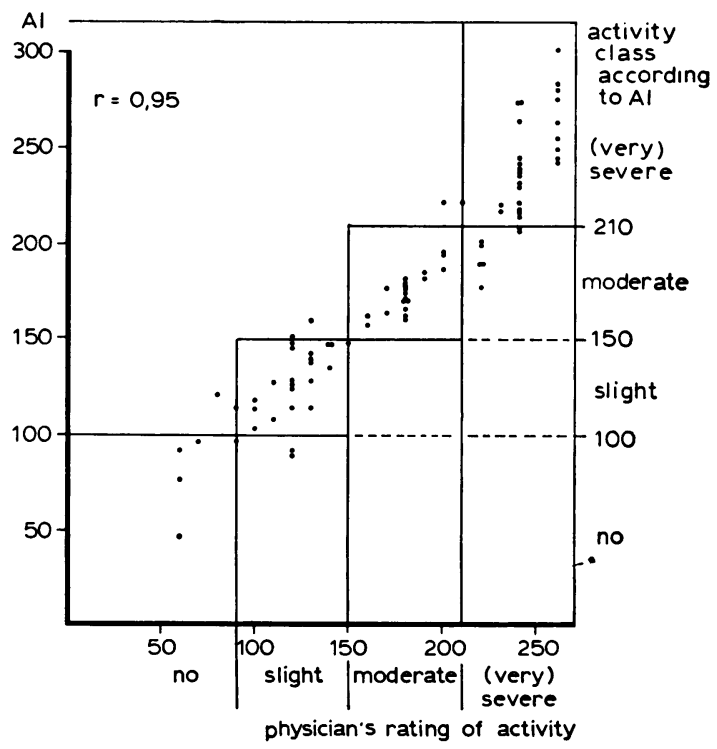

Fig. 1 Relation between the activity index (AI) and the physician's rating of inflammatory activity in 85 measurement sets (63 patients). 


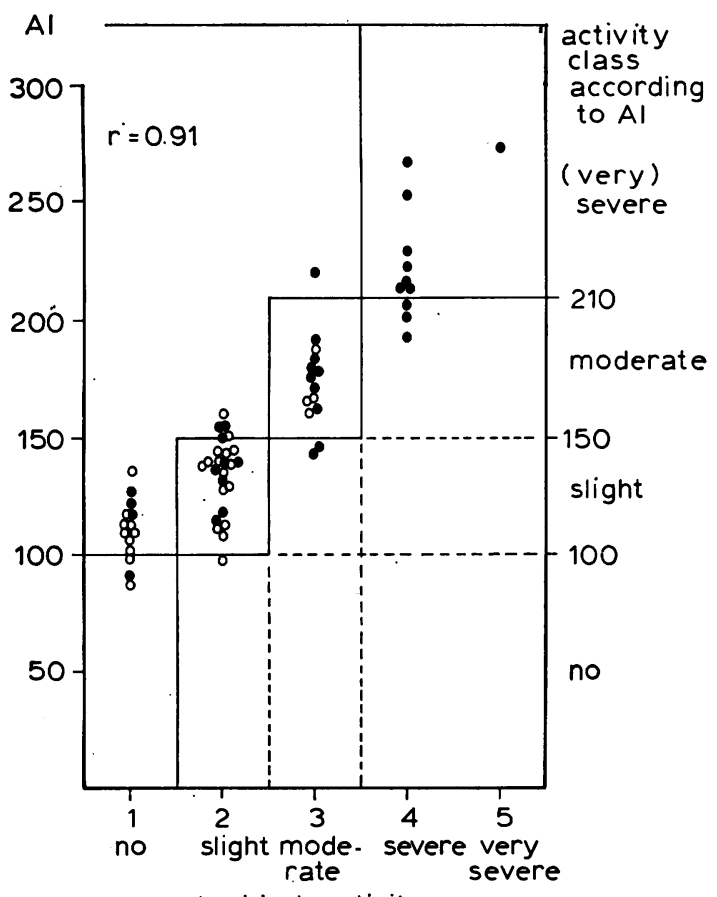

Fig. 2 Relation between activity index (AI) and physician's activity score in 34 clinical patients (O) and 30 outpatients (O) with Crohn's disease.

85 measurement sets are included in the same activity class by AI as by physician's rating.

EVALUATION OF AI AND CDAI

The correlation between the subjective ratings of the three gastroenterologists from other institutions and the AI was quite satisfactory: $0.74 ; 0.77$, and 0.87 , respectively.

In the 20 healthy volunteers the AI ranged from 41 to 116 points (mean \pm SD: $86 \cdot 1 \pm 18 \cdot 4$ ). Five $(25 \%)$ of these volunteers had an AI $>100$, which in patients with Crohn's disease would indicate slight activity. Values found for the CDAI ranged from -5 to 68 (mean \pm SD: $27 \cdot 0 \pm 20 \cdot 6$ ); according to Best et al. ${ }^{1}$ a CDAI $<150$ indicated quiescent Crohn's disease.

The relation between physician's rating and $\mathrm{AI}$ and CDAI, respectively, in the 64 patients with Crohn's disease is shown in Fig. 2 and 3, respectively. A good correlation was found between physician's rating and AI $(r=0.91)$. There was only a slight overlap in index values between successive classes of activity, with the exception of the classes 'no' and 'slight' activity. As Fig. 3 indicates, the correlation between physician's rating and CDAI was less pronounced $(r=0 \cdot 57)$. Many patients whose inflammatory activity was rated slight-to-moderate and even a few with severe activity, had a CDAI score within the same range as the CDAI in patients with quiescent Crohn's disease.

Figure 4 shows that only a moderate correlation $(r=0.67)$ existed between AI and CDAI in the 64 patients; if for both indices the boundary between no or slight activity and moderate or severe activity is set at 150 , then $34 \%$ of the patients are not identically classified according to both criteria.

The correlation between AI and physician's rating was also studied for some special groups in the series of 64 patients. In all these groups the correlation coefficient between AI and physician's rating was high: $r=0.91$ for 36 patients with Crohn's disease localised in the small intestine; $r=0.92$ for 25 patients with localisations in both the small intestine and the colon; $r=0.86$ for 22 patients who had undergone resection, and $r=0.91$ for 42 patients without resection.

Figure 5 indicates the $\mathrm{AI}$ and CDAI in 16 patients during the hospital period and at an outpatient follow-up 14 days later. In view of the short interval between the two observations the disease activity

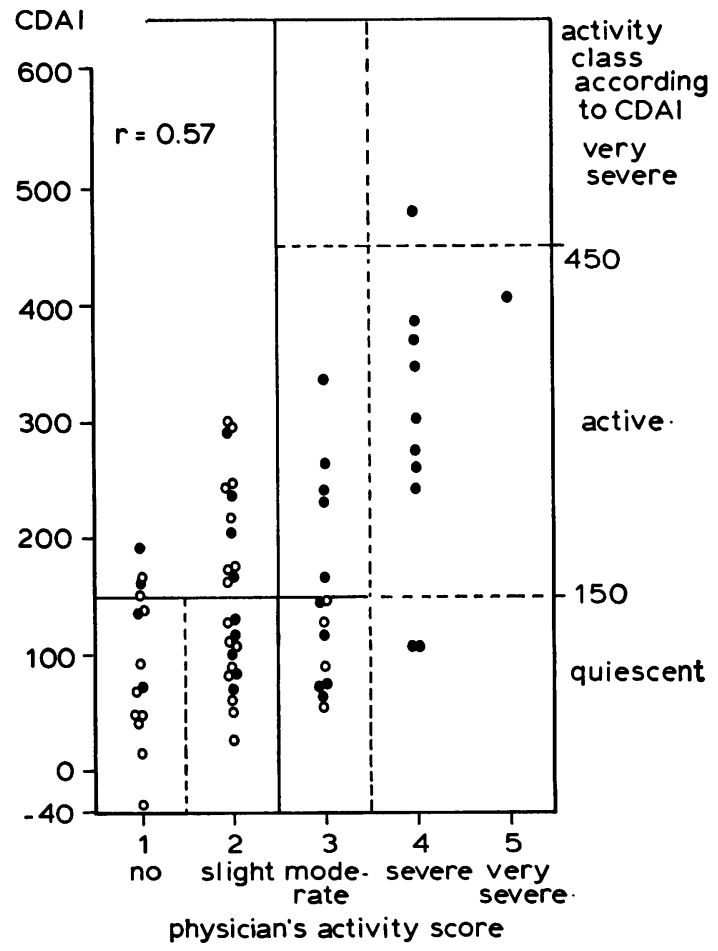

Fig. 3 Relation between CDAI and physician's activity score in 34 clinical patients (O) and 30 outpatients (○) with Crohn's disease. 


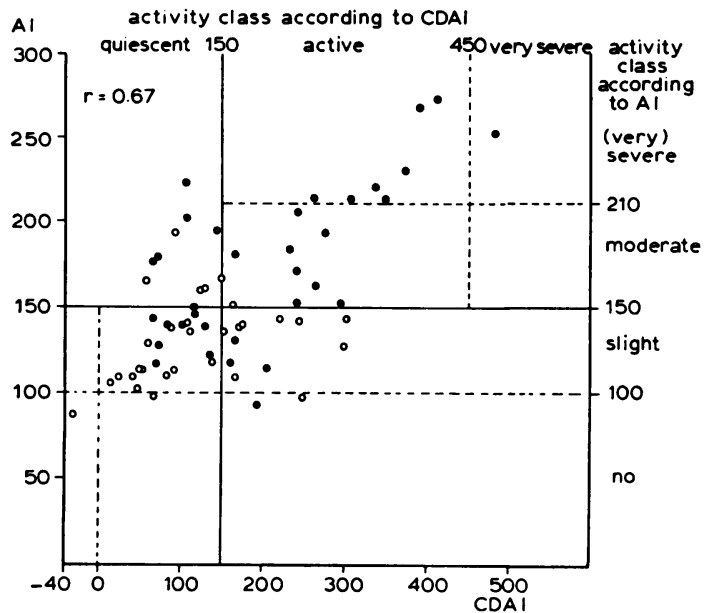

Fig. 4 Relation between AI and CDAI in 34 clinical patients (O) and 30 outpatients (O) with Crohn's disease.

was expected to show no significant increase or decrease. The AI was indeed found to have changed slightly (less than 20 points) in 12 of the 16 patients. In 11 of the 16 patients the physician's rating was the same on both occasions, and the AI was found also to have changed only slightly. In four patients the physican's rating at the follow-up was 1 point lower; in three of these four patients the AI was likewise
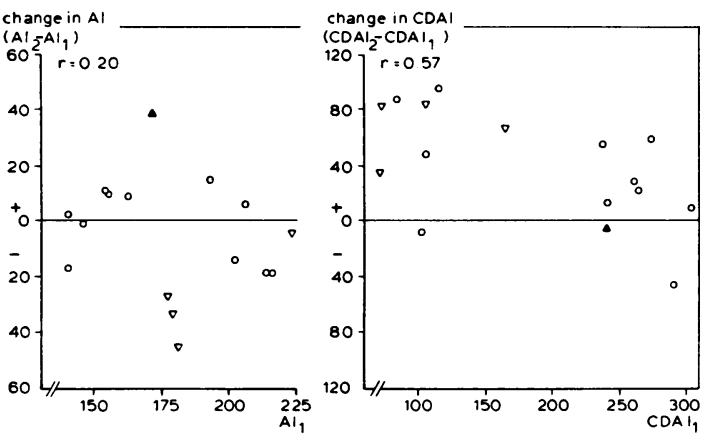

Fig. 5 Relation between the change in inflammatory activity after discharge from hospital and the inflammatory activity before discharge according to $A I$ and $C D A I$, in 16 patients with Crohn's disease $\left.\mathrm{AI}_{1} \mathrm{CI}_{1}\right\}$ activity before discharge.

$\left.\begin{array}{l}\mathrm{AI}_{2} \\ \mathrm{CDA} \mathrm{I}_{2}\end{array}\right\}$ activity at outpatient follow-up two weeks later. $\bigcirc$ Patients showing no change in physician's activity score. $\nabla$ Patients with one point decrease in physician's activity score. $\triangle$ Patients with one point increase in physician's activity score.

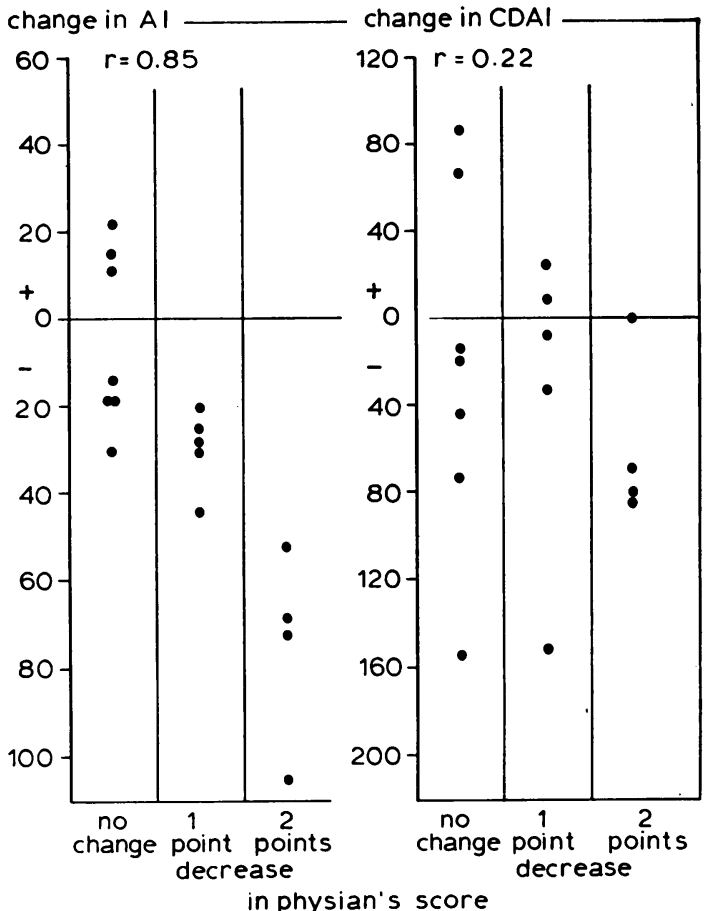

Fig. 6 Relation between the change in the activity indices ( $A I$ and CDAI) and that in the physician's activity score after one to six months of therapeutic medication in 16 patients with Crohn's disease.

substantially decreased. In one patient the inflammatory activity was rated 1 point higher, and the AI in this case showed a substantial increase.

The CDAI was substantially (more than 40 points) higher at the first outpatient follow-up than during the hospital period in eight of the 16 patients. Particularly in patients with a low CDAI in hospital, this increased considerably after discharge (even in patients in whom the physician's rating indicated decreased inflammatory activity after two weeks). This increase should probably be explained by an increase in subjective complaints after discharge from hospital. The CDAI is largely determined by subjective variables (general well being, abdominal pain).

The physician's subjective rating of the effect of therapeutic medication during one to six months on the inflammatory activity in 16 patients with Crohn's disease, and the corresponding activity indices AI and CDAI, are presented in Fig. 6. This Figure shows that the change in AI generally corresponded well with the physician's rating of the therapeutic effect. For the CDAI this was much less clear-cut. 


\section{Discussion}

Best et al. ${ }^{1}$ were the first to evolve a method of quantifying the inflammatory activity in Crohn's disease in a less arbitrary manner than was usually done in the past. The fact that this activity index (CDAI) is now being used in a number of prospective studies, demonstrates the urgent need for such an index. Nevertheless, the CDAI has a number of disadvantages. It was evolved with the cooperation of 18 physicians in 13 hospitals. At outpatient follow-up, these physicians formulated an overall evaluation of 'how the patient was doing'. At the same time, a large number of subjective and objective variables were registered. The number of patients thus studied totalled 112 (three to 20 patients from each centre). Multiple regression analysis was applied in an effort to determine which combination of variables correlated most closely with the subjective evaluation by the physician.

The publication of Best et al. ${ }^{1}$ does not indicate whether the physician based his rating on an overall subjective impression or in part also on data from the history, results of physical examination, or laboratory findings. The inter-rater agreement of the subjective ratings was not studied. In the development of the CDAI with the aid of multiple regression analysis, several variables were arbitrarily omitted (abdominal tenderness, serum albumin level) or added (body weight). The ultimate CDAI is made up of eight variables (stool consistency, abdominal pain, general well-being, extraintestinal symptoms of Crohn's disease, use of Lomotil or opiates against diarrhoea, abdominal mass, haematocrit, and body weight).

An important objection to the CDAI is that it is largely determined by subjective variables (abdominal pain, general well-being). The contribution of these variables to the sum of the standardised regression coefficients amounts to $39 \%$. To register these subjective variables, the patient must be asked to keep a diary card updated for at least a week. In the development of the CDAI, the fact that the medication often given in Crohn's disease may influence the subjective complaints (euphorising effect of prednisone, side-effects of salicylazosulphapyridine) was disregarded. Subjective complaints such as general malaise and abdominal pain are generally no reliable indication of inflammatory activity. Not infrequently, they result from complications or residual effects of the disease such as fistulisation or stenosis. The same applies to the rate of defaecation and stool consistency (their contribution to the CDAI amounts to 19\%). Patients with Crohn's disease who have undergone a bowel resection (specifically ileocaecal resection) not infrequently have a high defaecation rate and produce soft stools, even though there need be no question of any activity (or relapse) of Crohn's disease.

On the other hand, important objective parameters of inflammatory activity such as serum albumin level and ESR, have not been included in the CDAI. The validity of the CDAI is further reduced by the marked overlaps in CDAI score between the various activity classes established on the basis of subjective rating. The correlation coefficient between the physician's subjective rating and the CDAI calculated from the data supplied by Best et al. ${ }^{1}$ is only 0.70. In patients with Crohn's disease examined by us, the CDAI was found to change significantly without any change in the physician's rating of the disease activity (Fig. 5); also, the CDAI insufficiently reflected the effect of medication (Fig. 6).

The AI proposed in this paper, developed by analogy to the regression analysis applied by Best et $a .^{1}$ is made up almost entirely of objective variables (Table 4), of which the serum albumin level contributes most to the AI. The latter is explained by the fact that, according to the three specialists who rated the inflammatory activity, the serum albumin level is a good standard of activity in Crohn's disease. This opinion is based on the results of previous studies ${ }^{5}$ which showed a markedly higher correlation between serum albumin level and protein loss via the intestinal wall than between total serum protein, $\gamma$-globulin level, ESR, or haemoglobin concentration and protein loss via the intestinal wall. The authors hold that protein loss via the intestinal wall is the most reliable parameter to measure the inflammatory activity in the intestine. For practical reasons (relatively long time required, necessity of very careful collection of stools without admixture of urine, radiation load), however, this loss cannot be measured in all patients, and not repeatedly.

As the AI strongly depends on the serum albumin level this index will be affected by inter-laboratory differences in serum albumin determination. Correction for these differences as described above is necessary.

The variable 'sex' in the AI constitutes a correction on the contribution of the Quetelet index (the reference values of this index are lower in females than in males). ${ }^{23}$ The variable 'resection' is related to the variable 'stool consistency'. Patients with a history of resection often produce less well-formed stools, regardless of inflammatory activity.

The high correlation between physician's rating and AI $(r=0.95)$ is explained by the fact that the rating was done by three specialists of the same department, and was based exclusively on the 18 variables used in regression analysis. However, there 
was also a high correlation between the ratings of three gastroenterologists from other institutions and the AI ( $r=0.74,0.77$, and 0.87 , respectively). In the 64 patients whose inflammatory activity was rated by one of us, a high correlation between this rating and the $\mathrm{AI}$ was likewise found $(r=0.91)$, whereas the correlation with the CDAI was only $0 \cdot 57$. In this group of patients the rating was in part based also on other variables than those used in the development of the $\mathrm{AI}$-for example, data from the history, results of physical examination, and, sometimes, results of radiological examination. The high correlation between the physician's activity score and the AI, and the relatively low correlation with the CDAI, suggest that the physician's rating was based more on the characteristics included in the AI than on those contained in the CDAI. This has to be taken into account in comparing the merits of the two indices on the basis of Figs. 2, 3, 5, and 6, which is in favour of the AI. After all, this comparison is always based on a rating which was more AI- than CDAI-oriented. Nevertheless, the correlation between $\mathrm{Al}$ and rating $(r=0.91)$ was found to be considerably higher than that between the CDAI and the rating by the physicians involved in the construction of that index $(r=0 \cdot 70)$. Even the correlation between $\mathrm{AI}$ and the ratings of three gastroenterologists not involved in the development of our index was substantially higher $(r=0.74,0.77$, and 0.87 , respectively) than that between CDAI and the ratings of the physicians involved $(r=0 \cdot 70)$.

The results shown in Fig. 5 also argue in favour of the AI, in so far as the AI reflects less effect from discharge from hospital than the CDAl.

Figure 4 shows marked differences between the classification of the inflammatory activity of Crohn's disease according to the AI and the CDAI, respectively. This can be explained by the fact that the AI is based mostly on objective, and the CDAI substantially on subjective variables.
In view of the high correlations found between the AI and the physician's activity score within subgroups of the group of 64 patients, the AI can be used for patients with Crohn's disease localised in the small intestine as well as in the colon, and also for patients with a history of (limited) bowel resection. The AI shows an adequate response to changes in activity after therapeutic medication (Fig. 6).

Our findings would seem to warrant the conclusion that the AI is a reliable measure of inflammatory activity in patients with Crohn's disease, and is very suitable for evaluation of the effect of therapy in these patients.

The authors wish to thank Dr I Weterman (Leiden), Dr S Meuwissen (Amsterdam), and Dr A Bartelink (Groningen) for their contribution to the evaluation of the activity index, and $P$ Faaber for the determination of serum albumin both by cellulose acetate electrophoresis and radial immunodiffusion.

\section{References}

${ }^{1}$ Best WR, Becktel JM, Singleton JW, Kern F Jr. Development of a Crohn's disease activity index (National Cooperative Crohn's Disease Study). Gastroenterology 1976; 70: 439-44.

${ }^{2} \mathrm{Kh}$ sila, T, Lowe CT. Indices of obesity derived from body weight and height. Br J Prev Soc Med 1967; 21 : 122-8.

${ }^{3}$ Florey $\mathrm{C} \mathrm{du} \mathrm{V}$. The use and interpretation of ponderal index and other weight-height ratios in epidemiologic studies. J Chron Dis 1970; 23: 93-103.

${ }^{4} \mathrm{Nie}$ NH, Hull CH, Jenkins JG, Steinbrenner K, Bent DH, eds. Statistical package for the social sciences, 2nd ed. New York: McGraw Hill, 1975: 329-41.

${ }^{5}$ van Tongeren $\mathrm{JH}$, Eekhout A. Criteria to assess the severity of Crohn's disease and the effect of treatment. In: Weterman IT, Peña AS, Booth CC, eds. The management of Crohn's disease. Proceedings of the Workshop on Crohn's Disease, Leyden 23-25 October 1975. Amsterdam: Elsevier, 1976: 153-8. 